\title{
Online continuous detection of time-varying muscle synergies
}

\author{
Simone Ranaldi, Claudio Castellini, Andrea D’Avella, Silvia Conforto
}

\begin{abstract}
Muscle synergies have been widely used as a compact description of the neuromuscular motor control strategies. The online detection of synergistic activations might therefore improve the feasibility of sEMG-based control algorithms. In this study, a simple online detector of timevarying muscle synergies activation timings is proposed, and its performance is evaluated in a simulated online scenario on a small sample of experimental signals.
\end{abstract}

\section{INTRODUCTION}

$\mathrm{T}$ HE surface electromyography signal (sEMG) has been widely used to characterize the neural strategies underlying human motor control. The direct relationship between sEMG signals and neuromuscular control strategies has been often taken as a motivation to exploit those signals for improve the control of prostheses or virtual reality avatars [1]. Moreover, it is well known that the human musculoskeletal system has a redundant nature and that different muscles must be coordinated to achieve a variety of biomechanical functions. Thus, a physiologically inspired dimensionality reduction technique may significantly improve the outcomes of any real-time decision algorithm using the sEMG signal.

The coordination of different muscles is typically represented under the muscle synergy model; regardless of the physiological interpretation and mathematical implementation, the general feature of this model is that different muscles are acting in a patterned or grouped manner, i.e. as a synergy, to generate complex movements. Among the different muscle synergy models, the timevarying model is the one that describes muscle coordination in the most compact way [2]. Moreover, the combination of spatial and temporal information in the same synergy makes this description the most suitable for investigating the sEMG signal in a windowed way, potentially improving reliability of any sEMG-based decision algorithm. While the online processing of muscle synergies has been shown to be relatively straightforward when a synchronous model is exploited [3], the real-time detection of the time-varying synergistic structure is more complex and requires the optimization of different processing blocks.

In this work, a simple, projection-based method to

S. Ranaldi and S. Conforto are with the Department of Engineering, University of Roma Tre, Rome, Italy (corresponding author: S. Ranaldi, simone.ranaldi@uniroma3.it).

C. Castellini is with the Institute for Robotics and Mechatronics of the DLR - German Aerospace Center, Weßling, Germany.

A. D'Avella is with the Laboratory of Neuromotor Physiology, IRCCS Fondazione Santa Lucia, Rome, Italy and the Department of Biomedical and Dental Sciences and Morphofunctional Imaging, University of Messina, Messina, Italy. identify the activation of time-varying synergies starting from a pre-existent dictionary is presented and tested, with focus on its implications for online sEMG processing.

\section{METHODS}

\section{A. Time-varying muscle synergies}

The time-varying synergy model describes the coordination of several different muscles as the combination of the activity of a set of patterned muscle activations. In this description, a muscle synergy or motor module is defined as the potentially asynchronous activation, within a fixed time frame, of different muscles [2]. Considering this, any complex muscle activation pattern can be generated by the linear combination of a small number of synergies, optimally scaled in amplitude and shifted in time.

In the model of the time varying muscle synergy, each motor module is represented by a $\mathrm{N}_{\mathrm{m}} \times \mathrm{L}$ matrix, where $\mathrm{N}_{\mathrm{m}}$ is the number of muscles and $\mathrm{L}$ is the length of the synergies. If the muscle coordination is well represented by $\mathrm{N}_{\mathrm{s}}$ synergies, the sEMG envelope during a biomechanical event is then described by $\mathrm{N}_{\mathrm{s}}$ matrices determining the dictionary of synergies and two vectors of length $\mathrm{N}_{\mathrm{s}}, \mathrm{C}$ and $\tau$, indicating the scaling and timing coefficients.

\section{B. Experimental protocol}

The online synergy detector has been tested on upper limb reaching data coming from three subjects among those enrolled in [2]. All the details on the experimental protocol can be found in the referenced study. For this work, the kinematics of the movement was used only as a reference for the segmentation of the electromyographic signal. The sEMG envelopes, originally recorded at $1 \mathrm{kHz}$, have been downsampled at $100 \mathrm{~Hz}$.

\section{Data analysis}

The tonic component of the sEMG amplitude was estimated and removed from the signal, and time-varying synergies were then extracted following the same procedure adopted in [2]. To be coherent with the previous results on the same data, 5 synergies were extracted from all the subjects and the length of each module has been set to 500 $\mathrm{ms}$. The $\mathrm{R}^{2}$ value coming from the synergy approximation has been evaluated, and the $\mathrm{W}$ matrices were used as a dictionary for the online detector.

\section{Online detector}

The online detector works by projecting the first $200 \mathrm{~ms}$ of each synergy on the sEMG envelopes. At each time sample, all the synergies are projected onto the multi-muscle 
signal and the synergy with the greatest projection value after normalization is selected as a candidate for being active. The scalar product for that synergy is then evaluated for the subsequent windows until its maximum is reached; the beginning sample of this window is then fixed as the onset of the synergy and the scaling coefficient is set to the corresponding scalar product value. The synergy profile is then scaled by the amplitude coefficient and subtracted from the corresponding samples of the sEMG signal, similarly to what is done in the extraction algorithm [2]; moreover, when a synergy is found to be active it undergoes a refractory period for its entire duration. If at any time instant a different synergy has a higher scalar product with respect to the current candidate, that synergy is selected as the new candidate without defining any activation of the previous one; this criterion has been inserted to ensure that no spurious activations can be detected when no synergy is present, given that in presence of zero activity the projection values are randomly fluctuating.

The quality of the online detection has been quantified by using the estimated $\mathrm{C}$ and $\tau$ to reconstruct the original data and comparing the $\mathrm{R}^{2}$ coming from this approximation to the one found with the original synergy extraction algorithm. Moreover, the errors in the identification of the $\mathrm{C}$ and $\tau$ values have been computed as an additional metric.

\section{RESULTS}

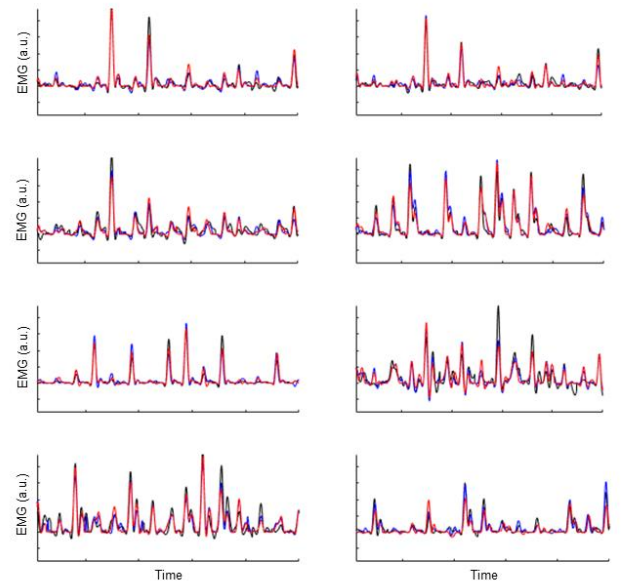

Fig.1: An example of the reconstruction of the original data (black) coming from the synergy extraction (blue) and from the online detector (red).

In order to ensure the validity of the results regardless of convergence to local minima, all the synergy estimation and subsequent projections have been replicated 30 times. $\mathrm{R}^{2}$ values coming from the original synergy extraction algorithm have been found to be $0.80 \pm 0.01$, while for the reconstructed data the quality of the reconstruction drops to $0.77 \pm 0.02$. On average, the online detector has an error of $5 \%$ of the original value for the amplitude coefficients and of $15 \mathrm{~ms}$ on the timing instants. From Fig. 1 an example of the comparison between the original data and the two reconstruction is shown on randomly selected subset of muscles for a 10 seconds time interval.

\section{DISCUSSION}

The presented results show that it is, in general, possible to build an algorithm for the online detection of the onset of the time-varying synergy from a multi-muscle sEMG recording. The small drop between the quality of the reconstruction from the optimization algorithm for synergy extraction and the one from the projection algorithm suggests that the performance of this kind of detector depends strongly on the quality of the synergy dictionary, that must be built on a subject-specific basis before the application of the controller. In this sense, future studies will have to investigate whether the dictionary built upon a relatively small portion of the time series is able to describe longer sequences of activations. In an ideal stationary model, if the calibration signal spans the entire movement space the synergy matrices are a complete basis for the SEMG space; however, several factors might affect the signal, potentially requiring a re-calibration of the dictionary. Among those factors, the co-adaptation mechanisms between the user and the controlled device might play a significant role and must be accurately characterized in a real life scenario.

A potential limitation of this online detector is the impossibility to account for activation of variable length; while in the literature some attempts to identify muscle synergies with a dynamic time warping approach have been proposed [4], the solution to the problem requires complex and computationally expensive algorithm that will detrimentally affect the real-time performance of the detector.

\section{CONCLUSIONS}

In this study, a projection algorithm for the online identification of time-varying synergies has been proposed. The results have shown that, if the synergy dictionary is well defined, it is possible to identify the onsets and the amplitude coefficients of the time-varying synergy starting from a window smaller than the length of the synergy itself. While some optimization procedures might be needed for a real-world implementation of this method, the results are promising in the framework of using these modular motor control concepts in myoelectric control algorithms.

\section{REFERENCES}

[1] C. Castellini, R. M. Bongers, M. Nowak, and C. K. van der Sluis, 'Upper-Limb Prosthetic Myocontrol: Two Recommendations', Front. Neurosci., vol. 9, 2016.

[2] A. d'Avella, A. Portone, L. Fernandez, and F. Lacquaniti, 'Control of Fast-Reaching Movements by Muscle Synergy Combinations', Journal of Neuroscience, vol. 26, no. 30, pp. 7791-7810, Jul. 2006.

[3] F. Lunardini, C. Casellato, A. d'Avella, T. D. Sanger, and A. Pedrocchi, 'Robustness and Reliability of Synergy-Based Myocontrol of a Multiple Degree of Freedom Robotic Arm', IEEE Transactions on Neural Systems and Rehabilitation Engineering, vol. 24, no. 9, pp. 940-950, Sep. 2016.

[4] Ramos, Felipe Moreira, Andrea d'Avella, and Mitsuhiro Hayashibe. "Identification of time-varying and time-scalable synergies from continuous electromyographic patterns." IEEE Robotics and Automation Letters 4.3 (2019): 3053-3058. 